\title{
openheart Trial protocol for the validation of the 'Toronto Aortic Stenosis Quality of Life (TASQ) Questionnaire' in patients undergoing surgical aortic valve replacement (SAVR) or transfemoral (TF) transcatheter aortic valve implantation (TAVI): the TASQ registry
}

Derk Frank, ${ }^{1}$ Simon Kennon, ${ }^{2}$ Nikolaos Bonaros, ${ }^{3}$ Mauro Romano, ${ }^{4}$ Thierry Lefèvre, ${ }^{4}$ Carlo Di Mario, ${ }^{5}$ Pierluigi Stefàno, ${ }^{5}$ Flavio Luciano Ribichini, ${ }^{6}$ Dominique Himbert, ${ }^{7}$ Marina Urena-Alcazar, ${ }^{7}$ Jorge Salgado-Fernandez, ${ }^{8}$ Jose Joaquin Cuenca Castillo, ${ }^{8}$ Bruno Garcia, ${ }^{9}$ Jana Kurucova, ${ }^{10}$ Martin Thoenes, ${ }^{11}$ Claudia Lüske, ${ }^{12}$ Peter Bramlage, ${ }^{12}$ Rima Styra ${ }^{13}$

- Additional material is published online only. To view please visit the journal online (http://dx.doi.org/10.1136/ openhrt-2019-001008).

To cite: Frank D, Kennon S, Bonaros N, et al. Trial protocol for the validation of the Toronto Aortic Stenosis Quality of Life (TASQ) Questionnaire' in patients undergoing surgical aortic valve replacement (SAVR) or transfemoral (TF) transcatheter aortic valve implantation (TAVI): the TASQ registry. Open Heart 2019;6:e001008. doi:10.1136/ openhrt-2019-001008

Received 9 January 2019 Revised 5 March 2019 Accepted 14 April 2019

Check for updates

(C) Author(s) (or their employer(s)) 2019. Re-use permitted under CC BY-NC. No commercial re-use. See rights and permissions. Published by BMJ.

For numbered affiliations see end of article.

Correspondence to Dr Derk Frank; derk.frank@ uksh.de

\section{ABSTRACT}

Background Patients with severe aortic stenosis (AS) have a reduced life expectancy and quality of life (QoL), owing to advanced age and the presence of multiple comorbidities. Currently, there is no AS-specific QoL measurement tool, which prevents an accurate assessment of how this chronic condition and its treatment affect patients. The Toronto Aortic Stenosis Quality of Life Questionnaire (TASQ) was developed in order to address this deficiency.

Methods The present trial protocol was designed to enable validation of the TASQ, which has been produced in five languages (English, French, German, Italian and Spanish) to increase usability. Patients with severe AS who are undergoing surgical aortic valve replacement (SAVR) or transcatheter aortic valve implantation (TAVI) will be asked to complete the TASQ and, for comparative purposes, the Kansas City Cardiomyopathy Questionnaire and the general health-related QoL Short Form-12 questionnaire. The questionnaires will be completed prior to the intervention, at discharge, as well as at 30 days and 3 months follow-up. A total of 290 patients will be recruited across one Canadian and nine European centres. Overall, the protocol validation aims to include 120 patients undergoing transfemoral TAVI (TF-TAVI), 120 undergoing SAVR and up to 50 being treated medically. The primary objective of the registry is to validate the TASQ in five different languages. The secondary objective is to assess the utility of the TASQ for assessing differences in QoL outcome between patients undergoing TF-TAVI, SAVR or medical management for their AS.

Discussion Validation and roll-out of the TASQ will enable clinicians to capture an accurate assessment of how AS and its management affects the QoL of patients and will help them to determine the most appropriate treatment strategy for individual patients.

\section{Key Questions}

What is already known about this subject?

- Patients with severe aortic stenosis (AS) have a reduced life-expectancy and quality of life, owing to advanced age andthe presence of multiple comorbidities.

- Currently, many different scales existto assess Quality of Life $(\mathrm{Q} o \mathrm{~L})$ in patients with cardiovascular disease. However, there is no AS-specific QoL measurement tool, which prevents anaccurate assessment of how this chronic condition and its treatment affect patients.

What does this study add?

- The Toronto Aortic Stenosis Quality of Life Questionnaire (TASQ) was developed in order to address this deficiency of anAS- specific QoL measurement tool.

- As with any novel questionnaire, validationof TASQ is required. In this protocol we describe the methods that will be used to validate the TASQ in patients with severe AS being treated with surgicalaortic valve replacement (SAVR), transcatheter aortic valve replacement (TAVI) or medical management.

Trial registration number NCT03186339

\section{INTRODUCTION}

Degenerative aortic stenosis (AS) is typically characterised by progressive calcification of the aortic valve, resulting in increased strain on the left ventricle. Once symptoms 


\section{Key Questions}

How might this impact on clinical practice?

- Validation and roll out of the TASQ will enable clinicians to capture an accurate assessment of how AS and its management affects the QoL of patients and will help them to determine the most appropriate treatment strategy for individual patients.

develop, the condition has usually reached such a severity that prompt intervention is essential. Without treatment, survival rates range from $15 \%$ to $50 \%$ at 5 years. ${ }^{1}$ Treatment with surgical aortic valve replacement (SAVR) has generally been the procedure of choice, while transcatheter aortic valve implantation (TAVI) has been developed as an alternative for patients who are at high or prohibitive risk for surgery. ${ }^{2}$ In some cases, an individual may be too sick or frail to undergo any form of invasive procedure. Such patients are treated with medication due to the limited chance of improvement after any form of surgical or interventional treatment.

Understanding how an illness can impact a patient's life is imperative to planning treatment strategies that will optimise the management of their symptoms and to determine the patient's satisfaction with the treatment they receive. ${ }^{34}$ While there is a great deal of information about survival and morbidity outcomes after aortic valve replacement, there is a lack of comprehensive data regarding quality of life (QoL) changes before and after the intervention. ${ }^{5-7}$ This is particularly significant in the setting of severe AS, where patients often have a reduced life expectancy, which affects their day-to-day physical and emotional condition. Furthermore, the cost of the procedure requires an assessment of the real value of the therapy taking into account the patient's and family perception of the benefit, that has an additional value.

It is important, therefore, to capture the physical and psychological disruptions caused by chronic diseases and their treatments. The level of interference varies by disease and by the different treatments received. ${ }^{8}$ Many different scales exist to assess QoL in patients with cardiovascular disease, including the Kansas City Cardiomyopathy Questionnaire (KCCQ) ${ }^{9}$ the Seattle Angina Questionnaire $(\mathrm{SAQ}),{ }^{10}$ the Minnesota Living with Heart Failure questionnaire ${ }^{11}$ and the MacNew heart disease health-related QoL questionnaire. ${ }^{12}$ There are also a number of generic health-related QoL questionnaires, including the widely used Short Form (SF)-12, ${ }^{13}$ the Euro-QoL five dimensions $(\mathrm{EQ}-5 \mathrm{D})^{14}$ and the Illness Intrusiveness Ratings Scale (IIRS). ${ }^{8}$ The KCCQ is perhaps the most relevant scale for application to patients with AS, as there are many similarities in the symptoms of AS to those of heart failure (HF). In a number of analyses of patients undergoing valve replacement, Arnold et al demonstrated a level of validity of the KCCQ. ${ }^{15-17}$ However, the treatment of AS differs substantially to that of HF, which significantly affects changes in QoL after any intervention.
In order to attain a more accurate picture of QoL for patients with severe AS before and after treatment, the Toronto Aortic Stenosis Quality of Life Questionnaire (TASQ) was developed. ${ }^{18}$ This questionnaire takes into account AS-specific symptoms and how they affect a patient's physical and mental well-being, as well as evaluating the patient's assessment of their general health. As with any novel questionnaire, validation of TASQ is required. Here, we describe the methods that we will use to validate the TASQ in patients with severe AS being treated with SAVR, TAVI or medical management. To facilitate dissemination of the questionnaire to multiple countries, it will be validated in five different languages using the standardised methodology described.

\section{METHODS AND ANALYSIS \\ Study design}

This is a prospective, multicentre, multinational registry with a follow-up period of 3 months. A total of 290 patients will be recruited across 10 centres, nine centres in Europe (Austria, ${ }^{1}$ France, ${ }^{2}$ Germany, ${ }^{1}$ Italy, $^{2}$ Spain $^{2}$ and the UK ${ }^{1}$ ) and one centre in Canada. Overall, this validation will include 120 patients undergoing transfemoral-TAVI (TF-TAVI) (Edwards Lifesciences, Irvine, California, USA), 120 patients undergoing (minimally invasive or full sternotomy) SAVR with any commercially available valve and up to 50 patients being treated medically. To avoid geographical or cultural variability, special attention will be payed on an even distribution of the patients enrolled within the centres. The TASQ has been produced in English and was validated in five languagesEnglish, French, German, Italian and Spanish—to ensure that patients can receive the questionnaire in their native language. Translation was conducted by members of all target countries including two forward and one backward translation steps followed by cognitive interviews on five patients with a heart condition and final proofreading. For each language, 58 patients will be recruited. For comparative purposes, patients will be required to complete the TASQ as well as the KCCQ and SF-12, at baseline, predischarge, as well as at 30 days and 3 months follow-up. The three questionnaires will be given to the patient sequentially, but in a random order.

To be included in the study, all patients need to be elective patients with severe symptomatic AS, have no major cognitive impairment and be able to provide written consent to participate in the study. Consent will be obtained by a study nurse at baseline, prior to TAVI/ SAVR procedure and after the patient has had a chance to read and understand the patient information leaflet provided.

\section{Development of the TASQ}

The TASQ was created by an interdisciplinary team of clinicians and patients with AS who were being assessed for TAVI procedures. Overall, 333 patients with AS were identified and interviewed to understand their current 
QoL and their expectations for the TAVI procedure, as well as their psychological coping, as part of their TAVI workup. Patients were asked to identify factors that they felt impacted their overall QoL. The factors were categorised as emotional, physical, symptoms or social limitations. Study participants' charts were reviewed to establish frequent themes of concern. Of the interviewed population, 211 patients underwent the TAVI procedure, 89 were declined for TAVI and 38 were still waiting for their TAVI at the time of chart review. Overall, patients had an average age of 80 years and $54.5 \%(n=115)$ of the patients were male.

Common physical symptoms identified were fatigue and shortness of breath, while completion of household chores and bathing were the most frequently identified physical limitations. Factors described as having an emotional impact included fear of a cardiac event, frustration with repeated admissions to hospital or visits to the emergency department, worry about maintaining their independence, inability to care for a dependent relative and distress that they were no longer able to plan for the future. The main social limitations included that the patient was no longer able to participate in social events or to socialise with friends as they had previously. The area of most concern was being able to meet with family members.

The prototype TASQ was assessed in a small study comprising 62 participants, using the KCCQ and IIRS scales for comparative purposes. The scoring of the TASQ was based on a consistent 7 point scale for each question, which covers response options from 'not very much' to 'very much'. Preliminary data showed that in this small number of patients there was good alignment of the TASQ with the KCCQ and IIRS scales. ${ }^{18}$ The final version of the TASQ questionnaire included a total of 16 questions from five domains (table 1). Each question has a maximum score of 7 , giving the complete questionnaire a maximum total score of 112 . The full questionnaire is provided in the (online supplementary file 1 ).

\section{Study objectives}

The primary objective of the registry is to validate the TASQ questionnaire in patients with severe symptomatic AS undergoing TAVI, SAVR or medical management

\begin{tabular}{lll}
\hline Table 1 A summary of the TASQ & \\
\hline Domain & Questions & Maximum points \\
\hline Physical symptoms & 1,14 & 14 \\
Physical limitations & $3,6,7,15$ & 28 \\
Emotional impact & $2,8,9,10,11,12,13$ & 49 \\
$\begin{array}{l}\text { Emotional impact and } \\
\text { expectations }\end{array}$ & 16 & 7 \\
Social limitations & 4,5 & 14 \\
& & Total score $=112$ \\
\hline
\end{tabular}

TASQ, Toronto Aortic Stenosis Quality of Life Questionnaire. in the five aforementioned different languages. The secondary objective is to assess the utility of the TASQ questionnaire for assessing differences in QoL outcome between patients undergoing TF-TAVI, SAVR or medical management for their AS. Further objectives are to compare the TASQ and its specific items with other non-dedicated questionnaires that are us in patients undergoing aortic valve replacement (ie, perception of autonomy, functional capacity, fear of dying, social life, etc). Further exploratory objectives include the assessment of the impact of the ejection fraction on the QoL in the context of the TASQ.

\section{Study population}

At each of the participating centres, patients will be consecutively enrolled if they have severe symptomatic AS and have been scheduled to undergo elective TF-TAVI or SAVR. Patients who are to be treated medically will be enrolled for comparison purposes. The treatment decision will be made by the heart team at each centre, based on standard in-house protocols, and will be independent of the study. Patients with major cognitive impairment and those who are unable to provide written informed consent will be excluded from the study.

\section{Data collection}

All data will be recorded on an electronic case report form (eCRF). At baseline, the eCRF will capture risk factors, pre-existing medical conditions, psychiatric disorders, ejection fraction and New York Heart Association class. Additional information for the eCRF includes answers of the TASQ KCCQ and SF-12 questionnaires (administered by research staff), patient demographic and clinical characteristics, employment status, living arrangements, activities of daily living and details of the procedure and postprocedural care (including plans for discharge and length of hospital stay) documented on the day of the TAVI/SAVR intervention. The questionnaires will be completed at baseline, predischarge, as well as at 30 days and 3 months follow-up. The schedule for data collection is given in table 2 .

The study nurse will receive a login and secret password on centre initiation to access the site and enter data. The eCRF has been designed to allow for automatic checks for plausibility and completeness. Data monitoring will be implemented by the sponsor. Two centres will be randomly selected, and approximately $20 \%$ of all patients will be assessed for accuracy.

\section{Statistical analysis}

The internal consistency of items in the TASQ will be measured using Cronbach's alpha. Construct validity will be correlated with the KCCQ. Floor effects will be calculated by comparing the number of patients scoring the worst possible score with the TASQ to the number of the patients scoring the worst possible score on the KCCQ. Ceiling effects will be calculated in the same manner. 
Table 2 Data collection schedule

\begin{tabular}{|c|c|c|c|c|c|}
\hline & Baseline & Intervention & Predischarge & 30 days & 3 months \\
\hline Patient information/informed consent & $X$ & & & & \\
\hline Demographics & $X$ & & & & \\
\hline Employment status, living arrangements, activities of daily living & $x$ & & & & \\
\hline Comorbidities* & $x$ & & & & \\
\hline Psychiatric disorders $†$ & $x$ & & & & \\
\hline Procedural details & & $\mathrm{X}$ & & & \\
\hline Procedural outcomes & & $x$ & & & \\
\hline Discharge plans, postprocedure length of stay & & $\mathrm{X}$ & & & \\
\hline Outcomes (MACCE) & & & $\mathrm{X}$ & $\mathrm{X}$ & $\mathrm{X}$ \\
\hline Anticipated further interventionsł & & & $\mathrm{X}$ & $\mathrm{X}$ & $x$ \\
\hline Delirium postprocedure & & & $x$ & $x$ & $x$ \\
\hline TASQ & $\mathrm{X}$ & & $\mathrm{X}$ & $\mathrm{X}$ & $x$ \\
\hline $\mathrm{KCCQ}$ & $x$ & & $x$ & $x$ & $X$ \\
\hline SF-12 & $X$ & & $X$ & $X$ & $X$ \\
\hline
\end{tabular}

*In particular, respiratory and cardiac conditions.

†Including present diagnosis of anxiety disorder or depression.

‡For example, transplant surgery.

KCCQ, Kansas City Cardiomyopathy Questionnaire; MACCE, major adverse cardiac and cerebrovascular events; SF-12, Short Form-12

questionnaire; TASQ, Toronto Aortic Stenosis quality of life Questionnaire.

Responsiveness and sensitivity to change before and after the TAVI/SAVR procedures and at the follow-up time points will be analysed with paired t-tests. Furthermore, t-tests will be used to compare baseline to predischarge, 30 days and 3 months outcomes and to compare 30 days to 3 months in terms of both QoL and patient expectations.

Changes between predischarge, 30 days and 3 months test results will measure responsiveness and sensitivity to change. Independent sample t-tests will be used to assess significant differences in the TASQ regarding QoL and symptom stability between the TF-TAVI and SAVR procedures.

Lastly, comparison analysis of QoL among the three QoL tools (TASQ, KCCQ and SF-12) and between TF-TAVI and SAVR patients will identify interactions between procedural group and measurement tool.

\section{DISCUSSION}

Patient QoL is increasingly being investigated as an outcome after the treatment of chronic disease. This is particularly relevant for patients with severe AS, who often have a short life expectancy. It is essential, therefore, that QoL be considered when weighing up the relative risks and benefits of the different strategies for managing AS.

Significant improvements in QoL have been demonstrated for patients with severe AS who undergo SAVR $^{19}{ }^{20}$ or TAVI. ${ }^{21-23}$ Arnold et al used the KCCQ, SF-12 and EQ-5D questionnaires to evaluate QoL in patients before and after SAVR and TAVI, and reported improvements in all three measures at 1, 6 and 12 months after postprocedure. ${ }^{15}$ An analysis of TAVI versus SAVR in the PARTNER trial found that KCCQ, SF-12 and EQ-5D scores improved after both procedures, with TAVI via TF access demonstrating a slight advantage over SAVR in the short term. ${ }^{24}$ In a second cohort of the placement of aortic transcatheter valves (PARTNER) trial, patients who were unsuitable for SAVR were randomised to TAVI or medical management. QoL was found to improve in both groups; however, the TAVI patients exhibited much greater improvements, with the difference between groups increasing over time. ${ }^{25}$ As the strongest improvement of the QoL was seen within the first week/months after the intervention, we opted for a 1 and 3-month follow-up in our project in an effort to keep the study design as pragmatic and focused as possible. We acknowledge the value of longer follow-ups, however.

While the questionnaires used in these studies to assess QoL in patients with severe AS have been demonstrated to have validity, they each have significant shortcomings. The KCCQ, although related to cardiac symptoms, is highly specific to HF, with every question reiterating this point. ${ }^{9}$ Furthermore, most questions refer to one or more HF-specific symptoms, including shortness of breath, fatigue and swelling of the ankles/feet. While these are also symptoms associated with severe AS, there are further factors that may affect the QoL of this elderly, multimorbid population. An important additional disadvantage of the KCCQ is the limited accuracy with which it can capture changes in QoL resulting from aortic valve replacement. Again, the questions are symptom specific, and do not encompass other more general QoL variables, such as mobility. They also fail to take into account the 
psychological implications prior to and after undergoing an intervention such as SAVR or TAVI.

On the other hand, the SF-12 refers to general health-related QoL. ${ }^{13}$ The only specific symptom that is mentioned is pain, which is unlikely to be among the most significant factors affecting the QoL in patients with severe AS. As patients with AS are generally elderly, many will have comorbidities and problems associated with frailty. ${ }^{26}$ In this setting, the SF-12 is not equipped to separate the effects of the AS from those of other conditions. The SF-12 has the advantage that it incorporates a number of general questions related to emotional and psychological issues associated with poor health; however, again, these are not specific to the AS patient population.

The TASQ was carefully designed in order to overcome the shortcomings of the currently available health-related QoL questionnaires. By incorporating insight from patients with severe AS, this novel assessment tool was developed to address the physical, emotional and social factors associated with this specific condition. The TASQ allows the patient to evaluate the effect of their "heart problems' on QoL, without specifying the symptoms that should form the basis for their conclusion. It includes a number of questions related to the implications of AS and its treatment on the patient's future; for example, concerns regarding family or finances.

An important element of the TASQ is the evaluation of changes in QoL that have occurred within the last 2 months. This allows for assessment of how the valve replacement itself has affected the patient's QoL. Completion of the questionnaires at discharge from hospital will be used to investigate any immediate changes in QoL resulting from the valve intervention, while those completed at 30 days and 3 months follow-up will provide an indication of the longer-term outlook after the procedure.

The ability to more accurately assess the QoL of patients undergoing treatment for severe AS will enable more appropriate treatment decisions to be made. Furthermore, it may help to identify specific concerns associated with the different therapies. These could then be addressed, for example, with rehabilitation, counselling or home care assistance.

\section{Potential limitations}

We elected to oversample due to attrition of patients during clinical trials. We also opted for a larger sample number to ensure stable data given that we were looking at cross language reliability and reproducibility. The sample size used non-probability sampling and was based on the envisaged recruitment rate during the enrolment period, which would allow for equal distribution of patient numbers across languages.

A further issue is that patients will be asked to complete three separate questionnaires, the inconvenience of which may result in missing answers. In order to spread the burden of this across the three forms, they will be administered to the patients in a random order. To address this possible problem, all patients will be evaluated for their compliance and ability to participate to the study in order to maximise the probability of complete follow-up.

Finally, we opted to go for TF-TAVI patients, as it is the standard access route in each of the centres. The documentation of non-TF patients would have a limited impact on clinical decision making (as TF-TAVI is standard) and would have potentially delayed recruitment substantially.

\section{CONCLUSION}

The TASQ was developed to address the absence of a QoL measure for patients with severe AS. The short life expectancy of this elderly, multimorbid population places greater emphasis on improving QoL, in contrast with the usual treatment aim of extending survival. It will also provide important information related to the patients and his family perception of the therapy value. This is essential in when the reimbursement of therapeutic options will be evaluated according to the patient satisfaction and perception of care value.

\section{Author affiliations}

${ }^{1}$ Departmentof Internal Medicine III (Cardiology, Angiology and Intensive Care Medicine), UKSH University Clinical Center Schleswig-Holstein, Kiel, Germany and DZHK (German Centre for Cardiovascular Research), partner site Hamburg/Kiel/ Lübeck, Kiel, Germany

${ }^{2}$ Department of Cardiology, Barts Heart Centre, St. Bartholomew's Hospital London, London, UK

${ }^{3}$ Departmentof Cardiac Surgery, Innsbruck Medical University, Innsbruck, Austria ${ }^{4}$ Departmentof Thoracic and Cardiovascular Surgery, Department of Interventional Cardiology, Hopital Privé Jacques Cartier, Massy, France

${ }^{5}$ Department of Structural Interventional Cardiology, Department of Cardiac Surgery, Careggi University Hospital, Florence, Italy

${ }^{6}$ Division of Cardiology, Department of Medicine, University of Verona, Verona, Italy ${ }^{7}$ Department of Cardiology, Bichat-Claude Bernard Hospital, Paris, France

${ }^{8}$ Department of Cardiology, Department of Cardiovascular Surgery, Hospital Juan Canalejo, Coruña, Spain

${ }^{9}$ Department of Cardiology, Hospital Vall d'Hebron, Barcelona, Spain

${ }^{10}$ Edwards Lifesciences, Prague, Czech Republic

${ }^{11}$ Edwards Lifesciences, Nyon, Switzerland

${ }^{12}$ Institute for Pharmacology and Preventive Medicine, Cloppenburg, Germany

${ }^{13}$ Department of Psychiatry, University Health Network, Toronto, Ontario, Canada

Acknowledgements Data were captured using the s4trials software provided by software for Trials Europe, Berlin, Germany.

Contributors DF, SK, TL, CL, PB and RS were involved in the conception and design of the registry. The remaining authors gave feedback on the final protocol and are currently including patients. DF, CL and PB drafted the manuscript and all other authors have been revising the article for important intellectual content. All authors have given final approval of the version to be published. All authors are fully accountable for the content of the manuscript.

Funding A research grant provided by Edwards Lifesciences (Nyon, Switzerland) to the Institute for Pharmacology and Preventive Medicine (Cloppenburg, Germany).

Competing interests $\mathrm{PB}$ is the representative of the Institute for Pharmacology and Preventive Medicine, Cloppenburg, Germany. All centres were paid by IPPMed for the enrolment and documentation of patients.

Patient consent for publication Not required.

Ethics approval The study protocol will be submitted to the relevant Independent Ethics Committee or Institutional Review Board at each centre for approval. The registry for this validation protocol will be in accordance with the Declaration of Helsinki and its amendments, as well as country-specific laws and regulations.

Provenance and peer review Not commissioned; externally peer reviewed.

Data availability statement There are no data in this work. 
Open access This is an open access article distributed in accordance with the Creative Commons Attribution Non Commercial (CC BY-NC 4.0) license, which permits others to distribute, remix, adapt, build upon this work non-commercially, and license their derivative works on different terms, provided the original work is properly cited, appropriate credit is given, any changes made indicated, and the use is non-commercial. See: http://creativecommons.org/licenses/by-nc/4.0/.

\section{REFERENCES}

1. Baumgartner H, Falk V, Bax JJ, et al. 2017 ESC/EACTS quidelines for the management of valvular heart disease. Eur Heart $J$ 2017;38:2739-91.

2. Leon MB, Smith CR, Mack M, et al. Transcatheter aortic-valve implantation for aortic stenosis in patients who cannot undergo surgery. N Engl J Med Overseas Ed 2010;363:1597-607.

3. Bonaros N, Petzina R, Cocchieri R, et al. Transaortic transcatheter aortic valve implantation as a first-line choice or as a last resort? An analysis based on the route registry. Eur J Cardiothorac Surg 2017:51:919-26.

4. Arnold SV, Spertus JA, Lei Y, et al. How to define a poor outcome after transcatheter aortic valve replacement: conceptual framework and empirical observations from the placement of aortic transcatheter valve (partner) trial. Circ Cardiovasc Qual Outcomes 2013;6:591-7.

5. Kundi $\mathrm{H}$, Strom JB, Valsdottir LR, et al. Trends in Isolated Surgical Aortic Valve Replacement According to Hospital-Based Transcatheter Aortic Valve Replacement Volumes. JACC Cardiovasc Interv 2018;11:2148-56.

6. Lancellotti P, Magne J, Dulgheru R, et al. Outcomes of patients with asymptomatic aortic stenosis followed up in heart valve clinics. JAMA Cardiol 2018;3.

7. Shi S, Afilalo J, Lipsitz LA, et al. Frailty phenotype and deficit accumulation frailty index in predicting recovery after transcatheter and surgical aortic valve replacement. The Journals of Gerontology: Series A 2018;62.

8. Devins GM. Using the illness intrusiveness ratings scale to understand health-related quality of life in chronic disease. $J$ Psychosom Res 2010;68:591-602.

9. Green CP, Porter CB, Bresnahan DR, et al. Development and evaluation of the Kansas City cardiomyopathy questionnaire: a new health status measure for heart failure. J Am Coll Cardiol 2000;35:1245-55

10. Spertus JA, Winder JA, Dewhurst TA, et al. Development and evaluation of the Seattle angina questionnaire: a new functional status measure for coronary artery disease. J Am Coll Cardiol 1995;25:333-41.

11. Rector TS, Cohn JN. Assessment of patient outcome with The Minnesota living with heart failure questionnaire: reliability and validity during a randomized, double-blind, placebo-controlled trial of pimobendan. pimobendan multicenter Research Group. Am Heart J 1992;124:1017-25.
12. Dixon T, Lim LLY, Oldridge NB. The MacNew heart disease healthrelated quality of life Instrument: reference data for users. Qual Life Res 2002;11:173-83.

13. Ware J, Kosinski M, Keller SD. A 12-Item short-form health survey: construction of scales and preliminary tests of reliability and validity. Med Care 1996;34:220-33.

14. EuroQol Group. EuroQol--a new facility for the measurement of health-related quality of life. Health Policy 1990;16:199-208.

15. Arnold SV, Reynolds MR, Wang K, et al. Health status after transcatheter or surgical aortic valve replacement in patients with severe aortic stenosis at increased surgical risk: results from the CoreValve us pivotal trial. JACC Cardiovasc Interv 2015;8:1207-17.

16. Arnold SV, Spertus JA, Lei Y, et al. Use of the Kansas City cardiomyopathy questionnaire for monitoring health status in patients with aortic stenosis. Circ Heart Fail 2013:6:61-7.

17. Arnold SV, Spertus JA, Vemulapalli S, et al. Quality-of-life outcomes after transcatheter aortic valve replacement in an unselected population: a report from the STS/ACC transcatheter valve therapy registry. JAMA Cardiol 2017;2:409-16.

18. Toronto Aortic Stenosis Quality of Life Scale (TASQ). Development and psychometric quality of life properties in TAVI patients. Toronto Aortic Stenosis Quality of Life Scale, 2017.

19. Folkmann S, Gorlitzer M, Weiss G, et al. Quality-of-life in octogenarians one year after aortic valve replacement with or without coronary artery bypass surgery. Interact Cardiovasc Thorac Surg 2010;11:750-3.

20. Oliveira SM, Correia AS, Paiva M, et al. Long-term survival, autonomy, and quality of life of elderly patients undergoing aortic valve replacement. J Card Surg 2012;27:20-3.

21. Biermann J, Horack M, Kahlert P, et al. The impact of transcatheter aortic valve implantation on quality of life: results from the German transcatheter aortic valve interventions registry. Clin Res Cardiol 2015;104:877-86.

22. Bona V, Khawaja MZ, Bapat V, et al. Early and late changes in quality of life following transcatheter aortic valve implantation using the transfemoral and transapical approaches. Eurolntervention 2015;11:221-9.

23. Fairbairn TA, Meads DM, Mather AN, et al. Serial change in healthrelated quality of life over 1 year after transcatheter aortic valve implantation: predictors of health outcomes. J Am Coll Cardiol 2012;59:1672-80.

24. Reynolds MR, Magnuson EA, Wang K, et al. Health-related quality of life after transcatheter or surgical aortic valve replacement in highrisk patients with severe aortic stenosis: results from the partner (placement of aortic transcatheter valve) trial (cohort a). J Am Coll Cardiol 2012;60:548-58

25. Reynolds MR, Magnuson EA, Lei Y, et al. Health-related quality of life after transcatheter aortic valve replacement in inoperable patients with severe aortic stenosis. Circulation 2011:124:1964-72.

26. Díez-Villanueva P, Salamanca J, Rojas A, et al. Importance of frailty and comorbidity in elderly patients with severe aortic stenosis. $J$ Geriatr Cardiol 2017:14:379-82. 